\title{
Análise comparativa entre regras heurísticas de sequenciamento da produção aplicada em job shop
}

\author{
José Arnaldo Montevechi, Dr. \\ João Batista Turrioni, Dr. \\ Dagoberto Alves de Almeida, PhD. \\ Ricardo Coser Mergulhão, Eng. \\ Fabiano Leal, Eng. \\ Programa de Pós-Graduação em Engenharia de Produção - Universidade Federal de Itajubá. \\ E-mail: layout@bol.com.br
}

\begin{abstract}
Este artigo apresenta a aplicação comparativa, através de um algoritmo desenvolvido, de quatro regras heurísticas de sequenciamento em job shop (SPT - Shortest Processing Time, LPT - Longest Processing Time, MWKR - Most WorK Remaining e LWKR - Least WorK Remaining). Esta aplicação é feita em uma empresa de deposição de partículas metálicas e cerâmicas. O trabalho demonstra que a eficiência de uma regra apresenta variação quando submetida a conjuntos de peças com diferentes faixas de tempo de processamento, segundo dois critérios analisados: tempo de ciclo e ociosidade dos equipamentos. No critério ociosidade, os tempos obtidos com a aplicação das regras apresentaram diferenças de até $100 \%$, enquanto que o critério tempo de ciclo apresentou diferenças de 17\%. A capacidade produtiva da empresa apresentou um aumento de 10,9\% após a adoção da regra MWKR.
\end{abstract}

Palavras chave: job shop; sequenciamento; regras heurísticas.

This article compares four Heuristic rules of schedule in job shop (SPT - Shortest Processing Time, LPT - Longest Processing Time, MWKR - Most WorK Remaining e LWKR - Least WorK Remaining) through a comparative application using an self developed algorithm. This application was accomplished in a company, which makes metallic and ceramic coating. Regards to the efficiency, different results were obtained when the rules were used at different range of processing time, according to two approachs: idleness equipment and cycle time. In the first one the times were different in up to $100 \%$ and the second one the results were different around $17 \%$. After the rule MWKR was applied the productive capacity of company has increased of $10.9 \%$.

Keywords: job shop; scheduling; heuristic rules.

\section{Introdução}

De acordo com Ichimura (1996), no ambiente de inovações tecnológicas constantes com diversidade de demanda e intensificação da competição no mercado, torna-se necessário diminuir os lotes de peças e adotar um sistema de ordens de produção, de modo a ordená-la conforme a demanda, em quantidades limitadas e com menor lead time.

Segundo Trentesaux et al. (2001), está clara, nos dias de hoje, a necessidade de uma programação inteligente da produção, devido sobretudo ao decréscimo do ciclo de vida do produto e o aumento da competição. Como conseqüência, um sistema de programação é agora avaliado não somente em termos de eficiência, mas também em termos de interatividade, de forma a integrar todas as necessidades dos gerentes em face ao alto número de restrições do processo.

Para atender às necessidades apresentadas, surgem técnicas de sequenciamento que permitem atingir soluções que proporcionam reduções de tempo de ciclo e menor ociosidade dos equipamentos, visando crescimento da capacidade produtiva e muitas vezes a redução de custos, de forma a tornar as organizações mais competitivas.

Segundo Lawrence e Sewell (1997), os métodos heurísticos surgem como um meio eficiente para atingir soluções aceitáveis sem grandes esforços computacionais. Além disso, os autores ainda afirmam que as seqüências ótimas, originárias de uma análise determinística, são deterioradas ao longo do tempo 
devido à presença de incertezas acerca do tempo de processamento em cada processo. Por outro lado, os autores consideraram os métodos heurísticos como comparáveis ou mesmo superiores aos métodos determinísticos, quando as incertezas do tempo de processamento estão presentes.

Conforme Trentesaux et al. (2001), o sistema de gerenciamento da produção é requisitado para elaborar, sobretudo, o sequenciamento da produção e o controle/avaliação do progresso da produção, a partir das seguintes premissas:

- Os produtos devem satisfazer às necessidades dos clientes em termos de qualidade, funcionabilidade, custo e tempo de entrega.

- Os sistemas de produção são caracterizados por restrições (tempo de produção, custo, etc.), além da capacidade limitada dos recursos.

Segundo Maccarthy e Liu (1993), o problema de programação pode ser definido, de um modo geral, como a alocação de recursos no tempo, de forma a executar um conjunto de tarefas. Programar a produção consiste em definir a ordem de entrada das tarefas a serem executadas na produção, ou seja, determinar como as tarefas devem ser conduzidas de uma máquina para outra.

De acordo com Montevechi e Pinho (1999), a programação da produção tem como um de seus objetivos maximizar a taxa de produção e minimizar o tempo total do fluxo de operação. Desta forma, uma troca na programação de operações pode resultar em economias na produção.

Uma ferramenta de representação de sequenciamento comumente utilizada é, segundo Slack et al. (1997), o gráfico de Gantt. Este gráfico indica quando cada trabalho está programado para começar e terminar. Uma vantagem do uso deste gráfico é a possibilidade de "testar” programações alternativas. Neste artigo, o gráfico de Gantt foi utilizado como base no algoritmo desenvolvido, para cada regra heurística analisada, a fim de se comparar os resultados obtidos.

De acordo com Zhou et al. (2001), o job shop consiste de um conjunto de $\boldsymbol{n}$ peças que são processadas em $\boldsymbol{m}$ máquinas, onde o processamento de cada peça consiste em $\boldsymbol{m}$ operações realizadas nestas máquinas em uma seqüência específica. Essa descrição segue a notação para problemas de job shop proposta por Graham et al. (1979), onde cada instância do problema é definida por um conjunto de peças, um conjunto de máquinas e um conjunto de tarefas ou operações. Segundo Baker (1974), o job shop tem a particularidade de não ser unidirecional.
Segundo Zhou et al. (2001), o sequenciamento em um ambiente job shop é um tópico importante na programação da produção. Seu foco está no ordenamento da entrada de ordens nas máquinas, de acordo com as restrições de processamento de cada peça, como os tempos de processamento, de modo a diminuir a duração do ciclo de processamento e, conseqüentemente, aumentar os ganhos financeiros.

De maneira geral, segundo Tarantilis e Kiranoudis (2002), as restrições presentes nas definições de sequenciamento em job shop são as seguintes:

- A precedência das tarefas para cada peça deve ser respeitada;

- Cada máquina pode desenvolver uma tarefa por vez;

- As operações não podem ser interrompidas.

O sequenciamento em job shop pode ser analisado sob a luz de algoritmos ou regras heurísticas, de forma a encontrar uma solução ótima ou sub-ótima para o problema, conforme os recursos disponibilizados.

Este artigo apresenta um estudo comparativo da eficiência de quatro regras heurísticas de sequenciamento (SPT - Shortest Processing Time, LPT - Longest Processing Time, MWKR - Most WorK Remaining e LWKR - Least WorK Remaining). A aplicação destas regras ocorreu no processo de uma pequena empresa de deposição de partículas metálicas e cerâmicas (job shop). Dois critérios foram utilizados para se identificar a regra mais eficiente: o menor tempo de ciclo e o menor tempo de ociosidade das máquinas. O trabalho também busca verificar se a eficiência de uma regra se mantém quando submetida a conjuntos de peças com altos e baixos tempos de operação.

O desempenho das regras é apresentado na forma de gráfico de barras, de acordo com o critério analisado. Um algoritmo foi desenvolvido para a aplicação das regras heurísticas no processo da empresa. A maneira pelo qual o algoritmo desenvolvido calcula os tempos de ociosidade e processamento, após aplicação de cada regra heurística, segue a idéia proposta pelo gráfico de Gantt. Esta representação está exemplificada na Tabela 2.

\section{Sequenciamento em Job Shop}

Segundo Zhou et al. (2001), é muito difícil alcançar uma solução ótima para o problema de job shop utilizando-se os métodos tradicionais de otimização, devido à sua alta complexidade computacional. 
Somente problemas de job shop envolvendo um número pequeno de máquinas e tarefas podem ser resolvidos com um tempo reduzido de análise computacional, com algoritmos que permitam obter respostas ótimas ao problema. (Carlier et al., 1989).

Zhou et al. (2001) ainda relatam a questão da solução exata como sendo não exigida em muitas situações práticas, isto porque o esforço computacional e os custos incorridos na obtenção de uma solução ótima exata não se compensam em muitas aplicações práticas de algoritmos de sequenciamento, onde soluções sub-ótimas são suficientes.

Existem métodos eficientes para procura de soluções sub-ótimas. Dentre eles, Rodammer e White (1988) destacam os métodos heurísticos, que têm sido objeto constante de pesquisas e que têm encontrado aplicação prática com resultados satisfatórios.

Ainda justificando o uso das regras heurísticas, Zhou et al. (2001) argumentam que a solução ótima em um problema de sequenciamento em ambiente job shop provém de um cálculo onde o número de interações é n!m , ao passo que, introduzindo regras heurísticas, o tamanho do espaço da solução seria reduzido para $\mathbf{n}^{\mathbf{m}}$. Neste operador, $\mathbf{n}$ corresponde ao número de tarefas a serem executadas e m corresponde ao número de máquinas onde as tarefas serão executadas. Tarantilis e Kiranoudis (2002) enfatizam esta questão, afirmando que, recentemente em seus trabalhos, resultados importantes foram alcançados através de métodos heurísticos. Estes resultados implicam em soluções satisfatórias obtidas com menor tempo de processamento.

Nos métodos heurísticos, o procedimento consiste em especificar uma regra de prioridade para selecionar uma operação entre várias operações candidatas a serem realizadas em uma mesma máquina. Através dos anos, regras de priorização de peças têm sido propostas por pesquisadores, tais como Blackstone et al. (1982), Haupt (1989), Ramasesh (1990) e Holthaus e Rajendran (1997). Segundo Houthaus e Rajendran (1997), não existe uma regra única que englobe simultaneamente critérios como, por exemplo, mínimo tempo de ciclo, mínimo número de peças em atraso e mínima ociosidade dos equipamentos. A escolha da regra depende de qual critério deseja-se melhorar.

Os critérios considerados neste estudo são os seguintes:

- Tempo de ciclo - corresponde ao tempo de processamento total da peça, de sua entrada no primeiro processo até sua saída no último processo.
- Ociosidade - representa o somatório de tempo em que o equipamento permanece à espera de uma determinada peça.

Quatro regras de priorização heurísticas - Shortest Processing Time (SPT), Longest Processing Time (LPT), Most WorK Remaining (MWKR) e Least WorK Remaining (LWKR) - foram selecionadas para inclusão neste estudo e apresentadas conforme citações na Tabela 1.

Tabela 1 - Descrição de regras heurísticas de priorização segundo alguns autores

\begin{tabular}{|c|c|c|}
\hline REGRA & DESCRIÇÃO & AUTORES \\
\hline $\begin{array}{l}\text { SPT } \\
\text { (Shortest } \\
\text { Processing } \\
\text { Time) }\end{array}$ & $\begin{array}{l}\text { Prioriza a peça com } \\
\text { o menor tempo de } \\
\text { processamento no } \\
\text { processo em } \\
\text { análise. }\end{array}$ & $\begin{array}{l}\text { Lawrence e Sewell (1997) } \\
\text { Baker (1974) } \\
\text { Zhou et al. (2001) } \\
\text { Conway et al. (1967) } \\
\text { Panwalker e Iskander } \\
(1977)\end{array}$ \\
\hline $\begin{array}{l}\text { LPT } \\
\text { (Longest } \\
\text { Processing } \\
\text { Time) }\end{array}$ & $\begin{array}{l}\text { Prioriza a peça com } \\
\text { o maior tempo de } \\
\text { processamento no } \\
\text { processo em } \\
\text { análise. } \\
\end{array}$ & Lawrence e Sewell (1997) \\
\hline $\begin{array}{l}\text { MWKR } \\
\text { (Most } \\
\text { WorK } \\
\text { Remaining) }\end{array}$ & $\begin{array}{l}\text { Prioriza a peça que } \\
\text { tem o maior tempo } \\
\text { de trabalho } \\
\text { acumulado nos } \\
\text { processos seguintes, } \\
\text { incluindo o corrente. }\end{array}$ & $\begin{array}{l}\text { Lawrence e Sewell (1997) } \\
\text { Baker (1974) } \\
\text { Zhou et al. (2001) } \\
\text { Conway et al. (1967) } \\
\text { Panwalker e Iskander } \\
(1977)\end{array}$ \\
\hline $\begin{array}{l}\text { LWKR } \\
\text { (Least Work } \\
\text { Remaining) }\end{array}$ & $\begin{array}{l}\text { Prioriza a peça que } \\
\text { tem o menor tempo } \\
\text { de trabalho } \\
\text { acumulado nos } \\
\text { processos seguintes, } \\
\text { incluindo o corrente. }\end{array}$ & $\begin{array}{l}\text { Lawrence e Sewell (1997) } \\
\text { Baker (1974) }\end{array}$ \\
\hline
\end{tabular}

Alguns autores compararam a aplicação de regras heurísticas segundo outros critérios. Correspondendo às afirmações de Houthaus e Rajendran (1997), cujo trabalho demonstrava que as regras de prioridade atuavam com melhores resultados para cada tipo de critério adotado, a regra SPT é, segundo Raghu e Rajendran (1993), a mais eficiente regra de minimização segundo o critério número de peças em atraso

Regras heurísticas foram examinadas por Conway et al. (1967), Panwalker e Iskander (1977) e Zhou et al. (2001). Estes autores concluíram que a regra MWKR foi a que apresentou os melhores resultados no critério minimização do tempo de ciclo. Em segundo lugar aparece a regra SPT, analisada segundo critério tempo de ciclo.

\section{Análise comparativa}

Os dados utilizados neste estudo comparativo (tempo de processamento de cada peça) foram obtidos em 
uma empresa que atua na área de deposição de partículas metálicas e cerâmicas em peças de diferentes tipos e formatos, atendendo a diferentes clientes.

O ambiente de produção da empresa pode ser classificado como um job shop, por apresentar um fluxo multidirecional, com as rotas das peças pré-definidas. O processo analisado consiste de quatro operações: preparação, jateamento, revestimento e acabamento, sendo que o acabamento e a preparação ocorrem nos mesmos equipamentos. Os dados aqui utilizados foram coletados no mês de março de 2002, em um dia típico de produção.

Devido à elevada amplitude de tempos de processamento, a empresa analisada classifica as peças em três grupos distintos, de acordo com o tempo de processamento total esperado para cada peça. Os grupos são:

- Baixo Tempo (BT): Tempo de processamento total de até 100 minutos.

- Médio Tempo (MT): Tempo de processamento total de 101 até 300 minutos.

- Alto Tempo (AT): Tempo de processamento total acima de 301 minutos.

Seguindo a definição adotada pela empresa, criaramse quatro grupos de 10 peças: Baixo Tempo (BT), Médio Tempo (MT), Alto Tempo (AT) e Misto. O grupo Misto é composto de peças dos três grupos anteriores, com a seguinte distribuição: 3 peças BT, 4 peças MT e 3 peças AT, com composição determinada através de sorteio aleatório dentro dos respectivos grupos.

A comparação realizada neste estudo envolve quatro regras de priorização (MWKR, SPT, LWKR e LPT) segundo dois critérios de análise: tempo de ciclo e ociosidade dos equipamentos. Cada regra heurística de priorização foi aplicada nos grupos de peças segundo a classificação fornecida pela empresa, para que se pudesse comparar a eficiência da regra heurística para peças com diferentes faixas de tempo de processamento.

O algoritmo utilizado para aplicação das regras foi desenvolvido na linguagem computacional Microsoft Visual Basic 5, tendo como softwares auxiliares o Access 7 e o Excel 7. O computador utilizado para os cálculos foi um Pentium 133 Mhz, com 32 Mb de memória RAM.

Conforme já apresentado, o algoritmo desenvolvido calcula os tempos de ociosidade e processamento, após aplicação de cada regra heurística, a partir da idéia proposta pelo gráfico de Gantt. A Tabela 2 exemplifica o processo de preenchimento da tabela pelo algoritmo desenvolvido, de acordo com a regra heurística analisada. Na Tabela 2, as colunas ( $\boldsymbol{j}$ ) estão divididas de acordo com as máquinas envolvidas no processo (neste exemplo, estão envolvidos dois tornos, duas fresas e duas retíficas). As linhas (i) representam o tempo gasto no processamento, em minutos. Os números marcados na tabela representam as peças trabalhadas no processo (neste exemplo, peça 1 e peça 2).

Cada lacuna preenchida da tabela $\mathbf{i} \mathbf{x} \boldsymbol{j}$ representa que, no tempo $\boldsymbol{i}$, a máquina j está ocupada com a peça (1 ou 2). O preenchimento da tabela depende do princípio da regra heurística selecionada, conforme demonstra a Tabela 1. Ao final do preenchimento da tabela, o algoritmo pode calcular os tempos de ciclo e ociosidade.

Tabela 2 - Exemplo da planilha preenchida pelo algoritmo, utilizando-se a idéia do gráfico de Gantt

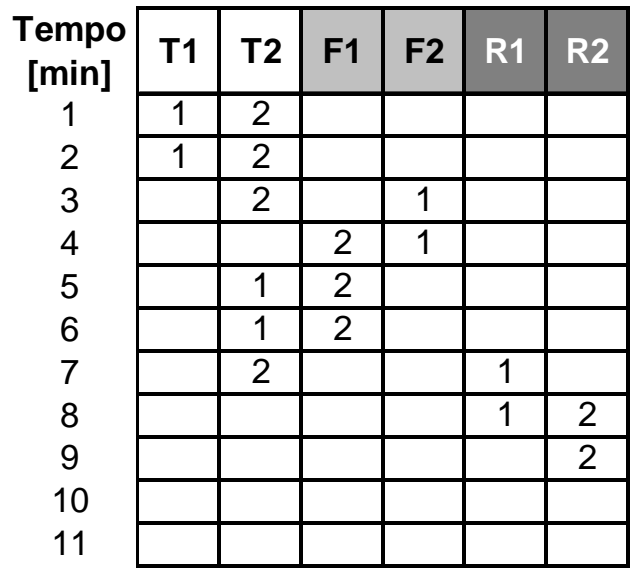

A Figura 1 apresenta os resultados da comparação entre as quatro regras heurísticas, associadas aos quatro grupos de peças, segundo o critério ociosidade.

Para peças de baixo tempo (BT), a regra LWKR apresentou a menor porcentagem de ociosidade (trata-se de um critério do tipo menor melhor), enquanto que a regra LPT apresentou-se como a de maior porcentagem de ociosidade, sendo a diferença cerca de duas vezes maior do que a regra LWKR. Nota-se ainda que, para peças do tipo BT, as regras baseadas na priorização do maior tempo (MWKR e LPT), apresentam-se menos eficientes, se comparadas às regras de priorização do menor tempo (LWKR e SPT).

No caso de peças de médio tempo (MT), a regra SPT foi a mais eficiente e a regra LPT a menos eficiente em termos de ociosidade. 


\section{Critério Ociosidade}

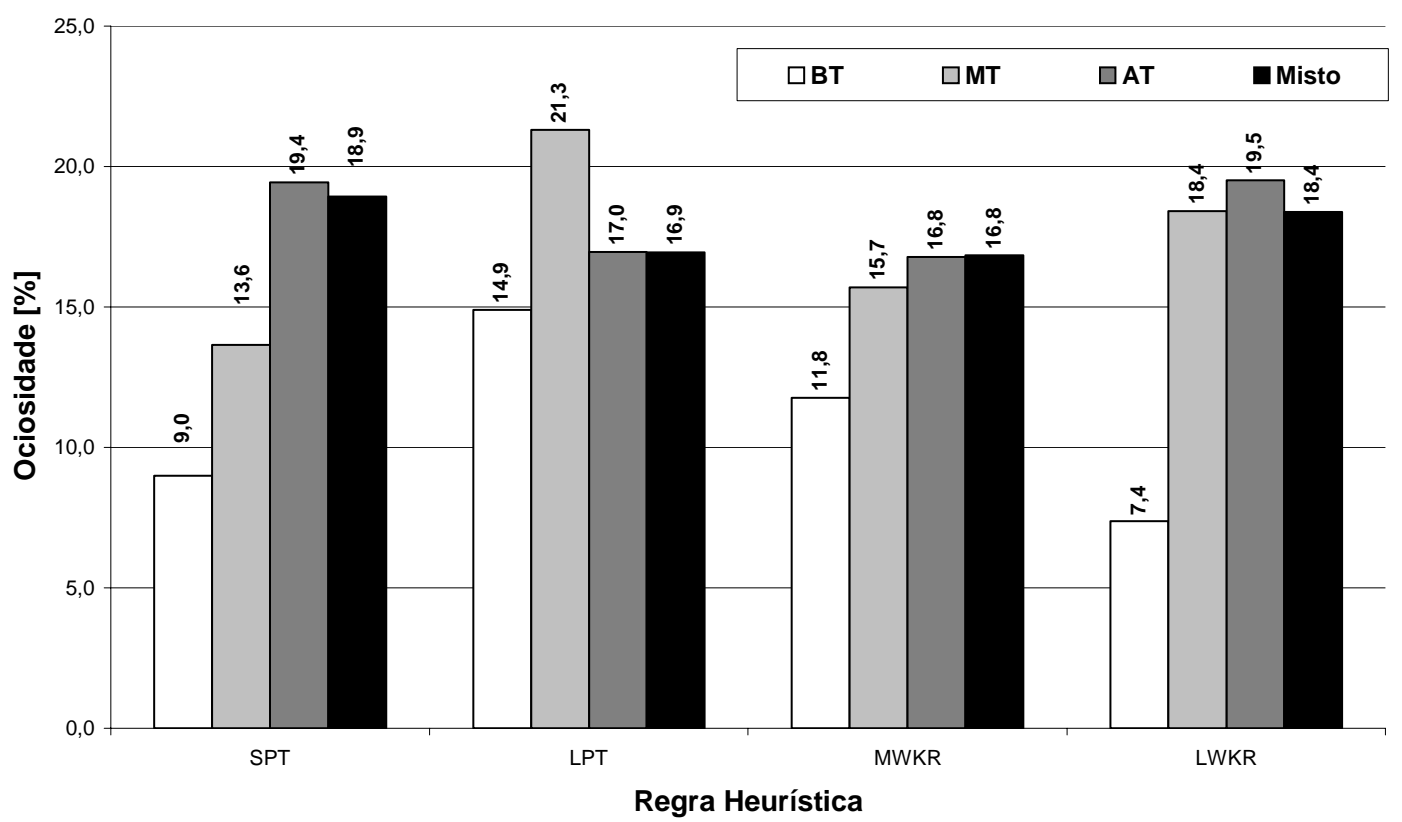

Figura 1 - Análise comparativa das quatro regras heurísticas segundo o critério ociosidade

No caso de peças de alto tempo (AT), observa-se um equilíbrio no desempenho das diferentes regras, sugerindo que a regra heurística escolhida para este tipo de peças tem pouca influência sobre o resultado. Observa-se uma pequena vantagem de desempenho da regra MWKR sobre as demais.

Para o caso de peças de tempo misto, os resultados apresentam equilíbrio semelhante àqueles obtidos para as peças do tipo AT.

Para simplificar a visualização dos resultados do critério ociosidade, foi construída uma matriz qualitativa $\boldsymbol{i}^{\prime} \boldsymbol{j}$, representada na Tabela 3, onde $\boldsymbol{i}$ representa a regra heurística (SPT, LPT, MWKR e LWKR) e $\boldsymbol{j}$ representa o grupo de peças analisado (BT, MT, AT e Misto). Na matriz, as regras são classificadas quanto ao desempenho, tal que o valor 1 é atribuído à regra mais eficiente e o valor 4 atribuído à regra menos eficiente. A coluna da média representa a média da pontuação atribuída a cada regra para seu respectivo conjunto. Dessa forma, a regra que apresentar menor pontuação média será a mais eficiente para o critério analisado. De acordo com a Tabela 3, a regra mais eficiente, levando-se em conta o critério ociosidade, é a MWKR.

A Matriz de resultados, representada na Tabela 3, também demonstra que a eficiência de cada regra analisada varia, quando submetida a grupos de peças com diferentes tempos de processamento. Aplicada a um grupo de peças com baixo tempo de processamen- to, a regra LWKR apresentou melhores resultados, de acordo com o critério ociosidade. Esta regra já não apresenta a mesma eficiência quando aplicada a um grupo de peças com um tempo alto de processamento, recebendo a ponderação 4, como mostra a Tabela 3. A mesma análise pode ser extrapolada às outras regras analisadas, que apresentaram variação de eficiência de acordo com o grupo de peças às quais foram submetidas.

Tabela 3 - Matriz de resultados para o critério ociosidade

\begin{tabular}{|c|c|c|c|c|c|}
\hline & BT & MT & AT & Misto & Média \\
\hline SPT & 2 & 1 & 3 & 4 & $\mathbf{2 , 5}$ \\
\hline LPT & 4 & 4 & 2 & 2 & $\mathbf{3 , 0}$ \\
\hline MWKR & 3 & 2 & 1 & 1 & $\mathbf{1 , 8}$ \\
\hline LWKR & 1 & 3 & 4 & 3 & $\mathbf{2 , 8}$ \\
\hline
\end{tabular}

Na Figura 2 apresenta-se os resultados da comparação entre as quatro regras heurísticas, associadas aos quatro grupos de peças, segundo o critério tempo de ciclo.

Para as peças de baixo tempo (BT), a regra MWKR apresentou-se como a mais eficiente no critério tempo de ciclo (quanto menor melhor). A regra MWKR fornece uma redução de $17,4 \%$ em cima do resultado de maior tempo de ciclo, que foi o SPT.

Para as peças de médio tempo (MT), a regra LWKR foi a mais eficiente para o critério tempo de ciclo. 
Critério Tempo de Ciclo

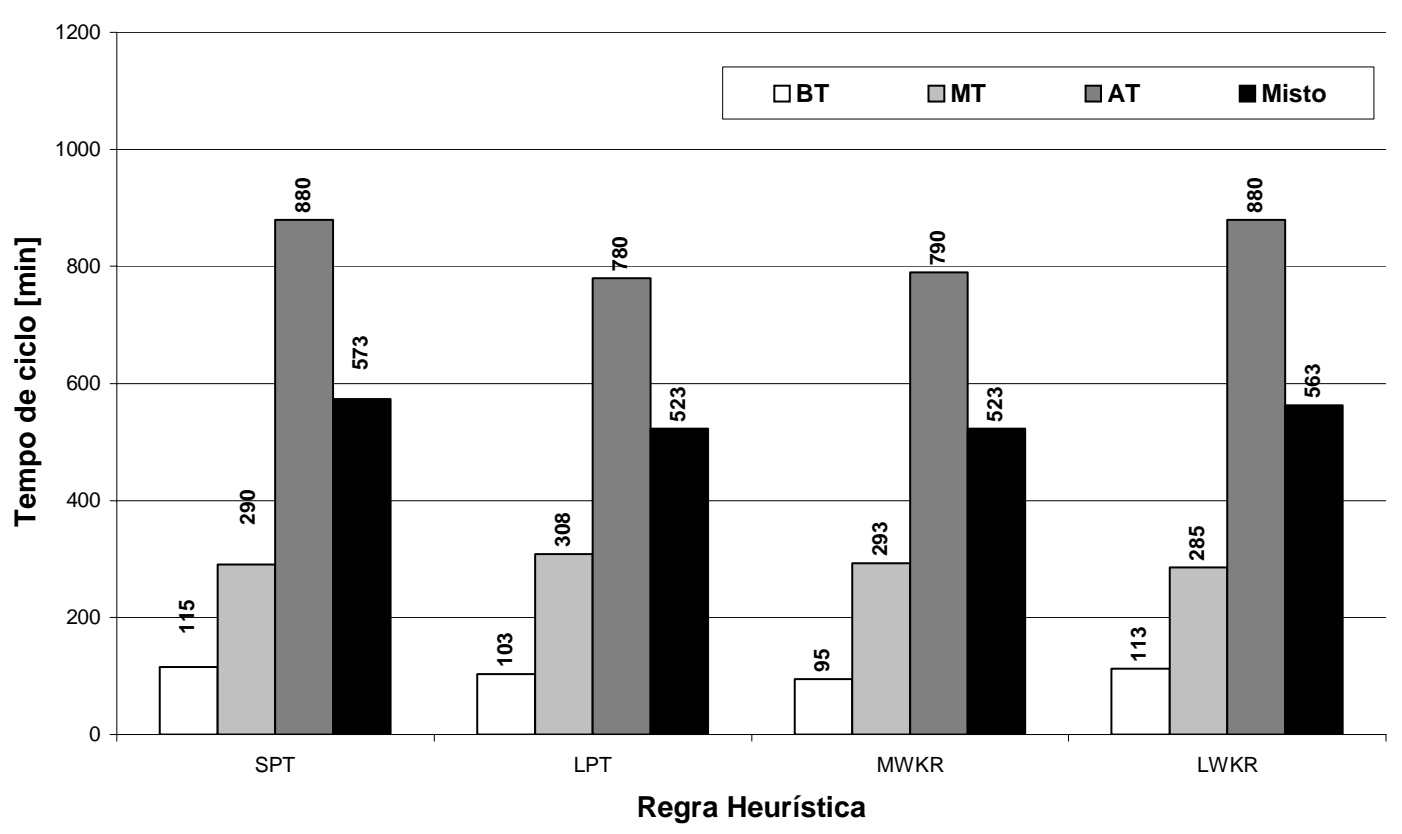

Figura 2 - Análise comparativa das quatro regras heurísticas segundo o critério tempo de ciclofoi a mais eficiente para o critério tempo de ciclo

Esta regra proporciona um tempo de ciclo 7,5\% menor do que o tempo de ciclo resultante da regra LPT.

Nas peças de alto tempo (AT) ocorreu um equilíbrio entre as regras LPT e MWKR quanto à melhor eficiência. Este equilíbrio também ocorre para as regras SPT e LWKR, que foram as menos eficientes, pois o tempo de ciclo se manteve cerca de $11,5 \%$ acima das regras de melhor eficiência neste caso.

Para as peças de tempo misto (Misto), as regras LPT e MWKR foram as mais eficientes, enquanto que as regras SPT e LWKR foram as menos eficientes, com uma diferença de 8,7\%.

A Tabela 4 traz uma matriz qualitativa de resultados similar à matriz representada na Tabela 3. De acordo com a Tabela 4, a regra mais eficiente, levando-se em conta o critério tempo de ciclo, é a MWKR.

Tabela 4 - Matriz resultados critério tempo de ciclo

\begin{tabular}{|c|c|c|c|c|c|}
\hline & BT & MT & AT & Misto & Média \\
\hline SPT & 4 & 2 & 3 & 3 & $\mathbf{3 , 0}$ \\
\hline LPT & 2 & 4 & 1 & 1 & $\mathbf{2 , 0}$ \\
\hline MWKR & 1 & 3 & 2 & 1 & $\mathbf{1 , 8}$ \\
\hline LWKR & 3 & 1 & 3 & 2 & $\mathbf{2 , 3}$ \\
\hline
\end{tabular}

Assim como analisado na Tabela 3, a Matriz de resultados representada na Tabela 4 também demonstra que a eficiência de cada regra analisada varia, quando submetida a grupos de peças com diferentes tempos de processamento. Segundo o critério tempo de ciclo, a regra LPT apresentou uma baixa eficiência quando aplicada a um grupo de peças com um tempo médio de processamento. Porém, ao ser aplicada a um grupo de peças com tempo alto de processamento, esta regra apresentou a melhor eficiência entre as regras comparadas.

\section{Conclusão}

Este artigo examinou quatro regras heurísticas para sequenciamento de ordens de produção em ambientes do tipo job shop. Considerando as matrizes qualitativas de resultados nas Tabelas 3 e 4, a regra MWKR foi a que promoveu os melhores resultados, segundo os critérios analisados. Porém, analisando-se os resultados individuais para cada grupo de peças, outras regras também se mostraram eficientes. Isto demonstra que a eficiência de cada regra não se mantém quando aplicada a conjuntos de peças com diferentes faixas de tempo de processamento. Estes resultados reforçam as conclusões de Zhou et al. (2001), como também as de Houthaus e Rajendran (1997).

No critério ociosidade, os tempos obtidos com a aplicação das regras apresentaram diferenças de até $100 \%$, enquanto que o critério tempo de ciclo apresentou diferenças de até $17 \%$ entre regras. Em termos de capacidade produtiva, a empresa, após adotar a regra de sequenciamento MWKR, registrou 10,9\% de aumento em sua capacidade produtiva, visto que havia mercado para absorver este aumento. 


\section{Referências}

BAKER, K. R. Introduction to Sequencing and Scheduling. New York: Johm Wiley \& Sons, 1974.

BLACKSTONE, J.H.; PHILLIPS, D.T. e HOGG, G. L. A state-of-the-art survey of dispatching rules for manufacturing job shop operations. International Journal of Production Research, v. 20, p.27-45, 1982.

CARLIER, J. e PISON, E. An algorithm for solving the job-shop problem. Management Science, v. 35, p. 167-176, 1989.

CONWAY, R.W.; MAXWELL, W.L. e MILLER, L.W. Theory of scheduling. New York: AddisonWesley, 1967.

GRAHAM, R.L.; LAWLER, E.L.; LENSTRA, J.K. e KAN, A.H.G. Optimization and approximation in deterministic sequencing and scheduling: A survey. ANAIS DA MATEMÁTICA DISCRETA, v. 5, p. 287-326, 1979.

HAUPT, R. A survey of priority rule-based scheduling. OR Spektrum, v. 11, p.3-16, 1989.

HOLTHAUS, O. e RAJENDRAN, C. Efficient dispatching rules for scheduling in a job shop. International Journal of Production Economics, v.48, p.87-105, 1997.

ICHIMURA, T. The elements and functions of hierarchical scheduling system of order production. International Journal of Production Economics, v.44, p.73-81, 1996.

LAWRENCE, S. T. e SEWELL, E. C. Heuristic, Optimal, Static, and Dynamic schedules when processing times are uncertain. Journal of Operations Management, v. 15, p. 71 - 82, 1997.

MACCARTHY, B. L. e LIU, J. Addressing the gap in scheduling research: a review of optimization and heuristic methods in production scheduling. International Journal of Production Research, v. 31, n. 1, p. 59-79, 1993.

MONTEVECHI, J. A. B. e PINHO, A. F. Incertezas em algoritmos de programação de operações. Artigo publicado nos ANAIS DO ENCONTRO NACIONAL DE ENGENHARIA DE PRODUÇÃO, Rio de Janeiro, 1999.

PANWALKER, S. S. e ISKANDER, W. A survey of scheduling rules. Operations Research, v.25, p.45-61,
1977.

RAGHU, T. S. e RAJENDRAN, C. An efficient dynamic dispatching rule for scheduling in a job shop. International Journal of Production Economics, v.32, p.301-313, 1993.

RAMASESH, R. Dynamic job shop scheduling: a survey of simulation research. Omega, v.18, p.43-57, 1990.

RODAMMER, F. A. e WHITE, K. P. A. A recent survey of production scheduling. IEEE Transactions on systems, v. 18, p.841-851, 1988.

SLACK, N.; CHAMBERS, S.; HARLAND, C.; HARRISON, A. e JOHNSTON, R. Administração da produção. 1.ed. São Paulo: Atlas S.A., 1997.

TARANTILIS, C. D. e KIRANOUDIS, C.T. A listbased threshold accepting method for job shop scheduling problems. International Journal of Production Economics, v.77, p. 159 - 171, 2002.

TRENTESAUX, D.; PESIN, P. e TAHON, C. Comparison of constraint logic programming and distributed problem solving: a case study for interactive, efficient and practicable job-shop scheduling. Computers \& Industrial Engineering, v. 39, p. 187-211, 2001.

ZHOU, H.; FENG, Y. e HAN, L. The hybrid heuristic genetic algotithm for job shop scheduling. Computers \& Industrial Engineering, v. 40, p. 191-200, 2001. 